\title{
Intraoperative Air Leak Test to Prevent Bile Leak After Right Posterior Sectionectomy with En Bloc Diaphragm Resection for Metastatic Teratoma
}

\author{
Timothy J. Vreeland, MD ${ }^{1}$, Eve Beaudry Simoneau, $\mathrm{MD}^{2}$, Whitney L. Dewhurst, $\mathrm{MS}^{1}$, Timothy E. Newhook, $\mathrm{MD}^{1}$, \\ Shannon N. Westin, $\mathrm{MD}^{3}$, Reza J. Mehran, $\mathrm{MD}^{4}$, Yun Shin Chun, $\mathrm{MD}^{1}$, Thomas A. Aloia, $\mathbf{M D}^{1}$, \\ Jean-Nicolas Vauthey, $\mathrm{MD}^{1}$, and Ching-Wei D. Tzeng, $\mathrm{MD}^{1}$ \\ ${ }^{1}$ Department of Surgical Oncology, The University of Texas MD Anderson Cancer Center, Houston, TX; ${ }^{2}$ Division of \\ Abdominal Transplant and Hepatobiliary Surgery, Department of Surgery, Columbia University, New York, NY; \\ ${ }^{3}$ Department of Gynecologic Oncology, University of Texas MD Anderson Cancer Center, Houston, TX; ${ }^{4}$ Department of \\ Thoracic and Cardiovascular Surgery, University of Texas MD Anderson Cancer Center, Houston, TX
}

\begin{abstract}
Background. The intraoperative air cholangiogram, or "air leak test" (ALT), at the time of hepatectomy can significantly reduce the rates of bile leak and symptomatic fluid collection after high-risk procedures. ${ }^{1,2}$ Because a bile leak in the setting of an en bloc diaphragm resection and mesh reconstruction would be a particularly dreaded complication, this video shows the technique for resection, reconstruction, and ALT.

Presentation. The video presents the case of a 29-year-old woman who had metastatic teratoma with an $8 \times 7-\mathrm{cm}$ liver metastasis in segment 7 and diaphragm invasion to the level of the right hepatic vein.

Operation. The authors performed a formal right posterior sectionectomy with en bloc diaphragm resection. The $12 \times 8$-cm diaphragmatic defect was reconstructed using biologic mesh (Surgimend, Integra LifeSciences, Plainsboro, NJ). An intraoperative ALT (air injection into the cystic duct with finger compression of the distal bile duct)
\end{abstract}

The video was presented at the Society of Surgical Oncology Annual Cancer Symposium March 2019 at San Diego, CA, USA.

Electronic supplementary material The online version of this article (https://doi.org/10.1245/s10434-019-07410-y) contains supplementary material, which is available to authorized users.

(C) Society of Surgical Oncology 2019

First Received: 22 January 2019;

Published Online: 7 May 2019

C.-W. D. Tzeng, MD

e-mail: CDTzeng@mdanderson.org identified several areas of bubbles from biliary radicles on the cut surface of the liver, which were ligated with 4-0 polypropylene. The ALT was repeated until no bubbles remained. Because no evidence of bubbles was observed, no surgical drain was needed. The patient did well postoperatively with no complications.

Conclusion. In cases of combined liver and diaphragmatic resection, prevention of bile leak, with subsequent contamination of the diaphragm repair and even the thoracic cavity, is particularly vital. An easily replicated intraoperative air leak test can mitigate the risk of bile leak and organ-space infection, as well as associated sequelae on quality of life, return to intended oncologic therapy, and oncologic outcomes.

DISCLOSURE There are no conflicts of interest.

\section{REFERENCES}

1. Aloia TA, Vauthey JN. Reply: air leak test: two hands are better than none. J Am Coll Surg. 2014;218:1078-9.

2. Zimmitti G, Vauthey JN, Shindoh J, et al. Systematic use of an intraoperative air leak test at the time of major liver resection reduces the rate of postoperative biliary complications. J Am Coll Surg. 2013;217:1028-37.

Publisher's Note Springer Nature remains neutral with regard to jurisdictional claims in published maps and institutional affiliations. 